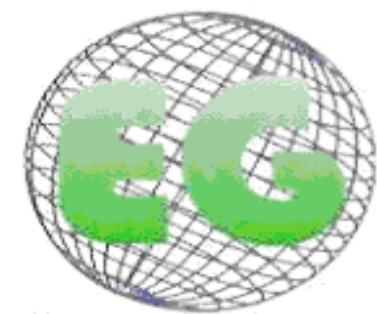

ISSN 1695-6141 N'22
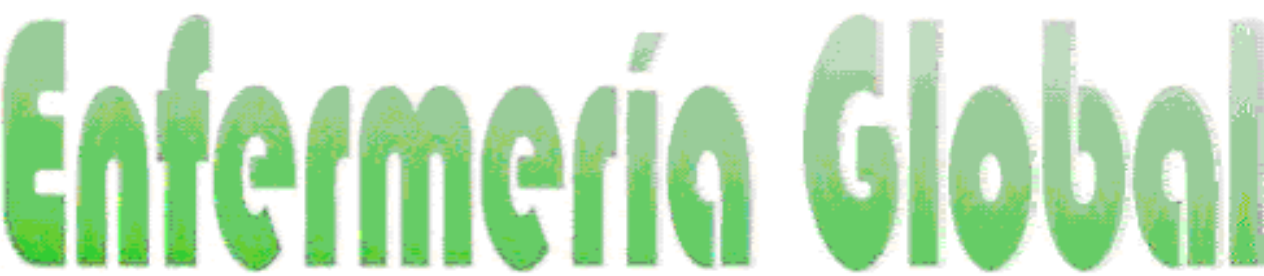

Revista electrónica trimestral de Enfermería

Abril 2011

www.um.es/egloball

REVISIONES

\title{
LA PRÁCTICA PEDAGÓGICA DEL DOCENTE EN ENFERMERÍA: UNA REVISIÓN INTEGRADORA DE LA LITERATURA
}

\section{A PRÁTICA PEDAGÓGICA PARA O DOCENTE EM ENFERMAGEM: UMA REVISÃO INTEGRATIVA DA LITERATURA}

*Sebold, LF., "* Carraro, TE.

\begin{abstract}
*Enfermera. Doctoranda del Programa de Pós Graduação da UFSC. Bolsista de CNPQ (Conselho Nacional de Desenvolvimento Científico e Tecnológico). "**Doctora en Enfermería. Profesora del Departamento de Enfermería y del Programa de Pós-Graduação da Universidade Federal de Santa Catarina. Brasil.
\end{abstract}

Palabras clave: Docente de Enfermería. Educación en Enfermería. Educación superior

Palavras-chave: Docente de Enfermagem; Educação em Enfermagem; Educação superior

Keywords: Nursing faculty; Nursing education; Higher education

\section{RESUMEN}

Se trata de una revisión integradora que tuvo como objetivo identificar en las publicaciones de revistas nacionales e internacionales indexadas en las bases de datos Lilacs, Cihnal, Medline, Scielo, Wilson, Bdenf en el período 2005-2009 los datos relacionados con el significado de la práctica pedagógica de los profesores de enfermería. Fueron obtenidos para el estudio 15 artículos. De los diversos enfoques y perspectivas de los docentes de enfermería sobre sus prácticas pedagógicas se mostró que los significados se relacionan con la experiencia en enseñar los conocimientos de enfermería, falta de preparación para ser profesor, entusiasmo, innovación y aprendizaje continuo en la carrera de enfermera-profesor. El mundo globalizado exige de los profesores la articulación de diversas competencias las cuales conforman la práctica de ser enfermera, la práctica pedogógica, la práctica docente y la formación pedagógica para que el proceso de enseñanza-aprendizaje alcance el éxito.

\section{RESUMO}

Trata-se de uma revisão integrativa que tem como objetivo identificar nas publicações de periódicos nacionais e internacionais indexadas nas bases Lilacs, Cihnal, Medline, Scielo, Wilson, Bdenf, no período de 2005-2009 dados relacionados ao significado da prática pedagógica para os docentes em enfermagem. Foram captados para o estudo 15 artigos. A partir das diversas abordagens e perspectivas dos enfermeiros docentes acerca de suas práticas pedagógicas evidenciou-se que os significados estavam relacionados à experiência em ensinar os saberes da enfermagem, despreparo para ser professor, entusiasmo, inovação e aprendizado constante na carreira de enfermeiroprofessor. O mundo globalizado exige dos professores a articulação de diversas competências as 
quais articulam a prática de ser enfermeiro, a prática pedagógica, a prática docente e a capacitação pedagógica para que o processo de ensino-aprendizagem alcance o sucesso.

\section{ABSTRACT}

This contribution is an integrating vision that seeks to identify publications in national and international journals indexed on the Lilacs, Cinnal, SCIELO, Wilson, Bdenf databases in the period 2005-2009 that are related to the significanc of the pedagogical practices of teachers of nursing. Data were obtained for the 15 subjects under study. Among the varying focuses and approaches of teachers of nursing towards their teaching practices the study found that significant issues are related to experience in teaching knowledge of nursing; not being oreoared to be a teacher; enthusiasm; innovation and continuous learning throughout one's career as a nurse and teacher. The globalized world demands that teachers have certain skills to perform the task of a nurse, to be a teacher and that they have the teacher training to successfully engage in the teacher-learner process.

\section{INTRODUCCIÓN}

Enfermería es una profesión reconocida mundialmente y legitimada por las personas como una práctica de cuidado para la preservación de la vida. Es una profesión que actúa en varias dimensiones, en el cuidado, en la investigación y en la educación. Y al actuar en la educación, el profesional enfermero se orienta para la carrera docente a través de la práctica pedagógica. La práctica pedagógica refleja las ideologías del contexto actuando de esta forma el proceso de la existencia humana ${ }^{(1)}$.

Se observa en el contexto de la enfermería brasileña que la práctica pedagógica viene siendo cada vez más repensada siguiendo los cambios de la globalización, teniendo en cuenta la busca constante de la aproximación de este mundo con las actividades de enseñanza-aprendizaje para que estas sean significativas para los sujetos del proceso educando y educador ${ }^{(2)}$.

Este repensar la práctica educativa posibilita la reflexión sobre la práctica docente, que va más allá de "preparar clases", obliga constantemente al profesor a pensar rectamente, y abarca también el movimiento dinámico, dialéctico, entre el hacer y el pensar sobre el hacer el cotidiano de enseñar-aprender. Configurando así el papel del profesor como un agente de transformación que contribuye positivamente a que el educando busque protagonizar su propia formación con el soporte necesario del educador ${ }^{(3)}$.

La práctica docente precisa ser crítica e implica que el profesor piense rectamente, abarcando el movimento dinámico, dialéctico entre el hacer y pensar sobre el hacer, o en otras palabras, el movimento continuo de la reflexión sobre el cotidiano del enseñaraprender $^{(3)}$.

Este movimento de aproximación representa una forma de educar, y está sujeta al desarrollo de la capacidad de reflexión de los enfermeros profesores, en la busca de una práctica docente que considere tanto los aspectos de capacitación en conocimientos y habilidades, como también basada en la ética y en el respeto a la autonomía de los educandos.

En este contexto, el enfermero que opta por actuar en la docencia precisa repensar sus prácticas de enseñanza del cuidado según sus propias formaciones y actuaciones para que puedan sentirse valorizados no solo como enfermeros, sino también como profesores de enfermería. 
Reflexionar sobre la práctica del enfermero profesor sea en el contexto internacional o nacional es un desafío, pues hacer comparaciones y constataciones en diferentes realidades en las que la educación en enfermería actúa en dinámicas distintas exige pensar en el contexto. Con estas reflexiones se buscó en la literatura la respuesta para la siguiente cuestión: ¿Cuáles son los significados de la práctica pedagógica para los docentes en enfermería?

Así, buscando elucidar los diferentes significados de la práctica pedagógica para los docentes en enfermería, este estudio tuvo como objetivo: identificar en las publicaciones de periódicos nacionales e internacionales indexadas en las bases Lilacs, Cihnal, Medline, Scielo, Wilson, Bdenf, en el periodo de enero de 2005 a diciembre de 2009, datos relacionados con el significado de la práctica pedagógica para los docentes en enfermería.

\section{METODOLOGÍA}

Se trata de un estudio de enfoque cualitativo que buscó identificar en las producciones científicas el significado de la práctica pedagógica para los docentes en enfermería. Se optó por una revisión integrativa de la literatura, siguiendo el modelo analítico propuesto por Ganong $(1987)^{(4)}$, pues este propicia el proceso de sistematización y análisis de los resultados dirigido a la comprensión de un determinado fenómeno a partir de estudios independientes.

La revisión integrativa abre la posibilidad de generar nuevos marcos y perspectivas sobre el fenómeno estudiado, así como la síntesis del estado de conocimiento de un determinado asunto, además de señalar lagunas del conocimiento que precisan ser completadas con la realización de nuevos estudios ${ }^{(5)}$. Además de esto, representa una herramienta de investigación valiosa para la Enfermería por permitir la síntesis de varios estudios publicados y extraer conclusiones generales sobre el área de estudio investigada. De esta manera, la revisión integrativa de la literatura es la revisión que busca evidenciar conclusiones en estudios anteriores resumiéndolos con la intención de formular conclusiones sobre un tópico específico $^{(4)}$.

Las etapas de esta revisión están establecidas bajo criterios formales y rígidos de investigación, desarrollándose a partir de los siguientes parámetros: selección de la pregunta de investigación; definición de los criterios de inclusión y selección de la muestra; representación de los estudios seleccionados en formato de tabla, considerando características comunes; análisis críticos de los resultados, identificando diferencias y conflictos; interpretación de los resultados; y reportar de forma clara la evidencia encontrada $^{(4)}$.

La selección del tema y la pregunta de pesquisa se apoyaron en la necesidad de identificación en la literatura, de la producción de conocimientos, acerca del significado de la práctica pedagógica para el docente en enfermería.

La estrategia de busca para identificación y selección de los estudios fue a través del estudio bibliográfico de publicaciones indexadas en las siguientes bases de datos: Literatura LatinoAmericana en Ciências de Saúde (LILACS); Cumulative Index to Nursing and Allied Health Literature (CINAHL); Literatura Internacional em Ciências da Saúde (MEDLINE); Scientific Electronic Library Online (SCIELO); WILSONWEB; Dados Bibliográficos na Área de Enfermagem do Brasil (BDENF). Las bases de datos MEDLINE, CINAHL, WILSONWEB fueron accesadas a través de los links disponibilizados por la Biblioteca Universitária da 
Universidade Federal de Santa Catarina (BU/UFSC) link Portal da Capes, en la dirección electrónica <http://www.bu.ufsc.br/>. Las bases de datos LILACS, SCIELO, BDENF, fueron accesadas a través del portal de la Biblioteca Virtual da Saúde (BVS), disponible en el sitio <http://www.bireme.br/php/index.php>. Los descriptores fueron selecionados a partir de la terminología en salud consultada en el National Library of Medicine's (MESH) y en los Descriptores em Ciências da Saúde (DECS-BIREME), y son: Faculty, nursing; Nursing faculty practice; Teaching; Education; Education, higher; Education, nursing.

La investigación en las bases de datos se realizó entre los meses de abril, mayo y junio de 2010, y los estudios encontrados en más de una base de datos fueron considerados solamente una vez, excluyéndose los artículos duplicados. Los criterios adoptados para la selección de los artículos fueron: categorías de artículo (original, revisión de literatura, reflexión, relato de experiencia); artículos con resúmenes y textos completos; disponibles en los idiomas portugués, inglés o español; publicados entre los años 2005 a 2009, y artículos que contengan en sus títulos y/o resúmenes indicativos que muestren los significados de la práctica pedagógica para el docente en enfermería. El recurso utilizado en la investigación fue la opción "término exacto" durante la realización de las búsquedas. Los criterios de exclusión de los artículos fueron los estudios que no atendieron a los criterios de inclusión mencionados arriba, las producciones duplicadas, propagandas, cartas y editoriales así como los artículos que abordasen otras temáticas diferentes del objetivo del estudio.

La busca por producciones resultó inicialmente en los siguientes números en las referidas bases: LILACS: 43 artículos; CINAHL: 436 artículos; MEDLINE: 38 artículos; SCIELO: 57 artículos; WILSONWEB: 509 artículos; BDENF: ningún artículo encontrado. Totalizando en esta etapa del estudio la cantidad de 1083 artículos.

Tras la captura de todos los artículos se pasó a la lectura de los títulos y resúmenes considerando que de esta forma se seleccionarían los artículos que presentasen adherencia con el objetivo propuesto. Quedando entonces 108 artículos que fueron leídos en la íntegra, siendo todavía excluídos 93 y permaneciendo para el estudio 15 artículos. Estando 3 artículos en la base LILACS; en la base CINAHL 2 artículos; MEDLINE 3 artículos; SCIELO 4 artículos; MILSONWEB 3 artículos.

Tabla 1: Distribución de los estudios según las Bases de Datos y criterios de inclusión y exclusión, en el período de abril a junio, SC, 2010.

\begin{tabular}{l|c|c|c|c}
\hline Base de Datos & $\begin{array}{c}\text { Número } \\
\text { artículos }\end{array}$ & $\begin{array}{c}\text { Número de } \\
\text { artículos por } \\
\text { refinamiento } \\
\text { (título y abstract) }\end{array}$ & $\begin{array}{c}\text { Número de } \\
\text { artículos } \\
\text { relevantes } \\
\text { (artículos } \\
\text { completos) }\end{array}$ & $\begin{array}{c}\text { Número de } \\
\text { artículos excluídos }\end{array}$ \\
\hline LILACS & 43 & Incluído & Incluído & 16 \\
\hline CINAHL & 436 & 9 & 3 & 3 \\
\hline MEDLINE & 38 & 6 & 2 & 18 \\
\hline SCIELO & 57 & 22 & 3 & 50 \\
\hline WILSONWEB & 509 & 53 & 3 & 6 \\
\hline
\end{tabular}




\begin{tabular}{l|c|c|c|c}
\hline BDENF & 0 & 0 & 0 & 0 \\
\hline TOTAL & 1083 & 108 & 15 & 93 \\
\hline
\end{tabular}

Esta tabla fue construida a fin de poner en práctica y cuantificar los criterios de inclusión y exclusión aplicados al realizarse las etapas de lectura de título, resumen y texto completo, respectivamente, hasta que se alcanzase el objetivo de la investigación. El total de quince artículos representa específicamente los datos que responden a la pregunta de la investigación, que fue identificar el significado de la práctica pedagógica para el docente en enfermería.

En la etapa de organización y evaluación de los estudios incluidos en la revisión, tras la selección, los estudios fueron archivados en carpetas en el directorio del computador. Los trabajos considerados relevantes fueron rescatados en forma completa y guardados en carpetas por año de publicación. Los materiales seleccionados fueron dispuestos en planilla del Programa Excel for Windows, de Microsoft, con las siguientes informaciones: base de datos, título del artículo, periódico, año, país de origen, categoría del estudio, natureza del estudio, referencial teórico, método de análisis, población del estudio, objetivo del estudio, enfoque del tema, autores (titulación y formación). De esta manera los datos fueron agrupados conforme la identificación de semejanzas y diferencias constituyendo un hallazgo que fue interpretado y analizado con literaturas pertinentes.

\section{RESULTADOS}

Para analizar y discutir la temática del significado de la práctica pedagógica para el docente en enfermería se procedió, inicialmente, a la tabulación de los datos en relación al año de publicación y los países de origen. Partiendo del análisis del año de publicación de los artículos se verifica que la mayoría de las publicaciones se dio en los años 2006, 2008 y 2009, teniendo como mayoría los artículos de origen brasileño. Los resultados evidencian que las informaciones en los diferentes escenarios de enfermería presentan características en común.

Gráfico 1 - Distribución de los estudios según el origen, em el período de abril a junio, SC, 2010.

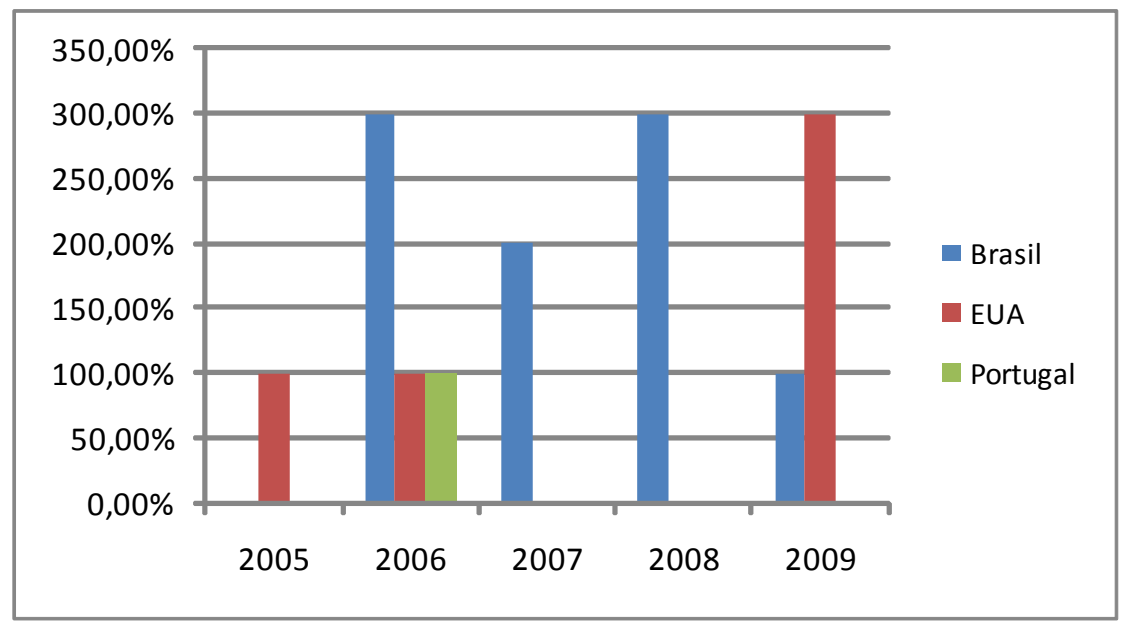

Es un hecho que de las 15 producciones científicas, seis se publicaron en la Revista Brasileira de Enfermagem - REBEN; cuatro en el Journal of Nursing Education que es específica del área de educación en enfermería y tiene como país de origen los Estados 
Unidos de América (USA); tres en la Revista da Escola de Enfermagem da Universidade de São Paulo; una en Medsurg Nursing; una en la Revista Latino Americana de Enfermagem; una en The Journal of Continuing Education in Nursing.

La siguiente tabla presenta la base de datos, el título, el periódico, año y país de origen del artículo:

\begin{tabular}{|c|c|c|c|c|}
\hline $\begin{array}{l}\text { Base de } \\
\text { datos }\end{array}$ & Título del artículo & Periódico & Año & País \\
\hline LILACS & $\begin{array}{l}\text { Obstáculos didáticos no cotidiano da } \\
\text { prática pedagógica do enfermeiro } \\
\text { professor }\end{array}$ & $\begin{array}{l}\text { REBEn (Revista } \\
\text { Brasileira de } \\
\text { Enfermagem) }\end{array}$ & 2008 & Brasil \\
\hline LILACS & $\begin{array}{l}\text { A prática de ensinar: dialogando com as } \\
\text { professoras de enfermagem }\end{array}$ & REBEn & 2008 & Brasil \\
\hline LILCAS & $\begin{array}{l}\text { A prática pedagógica das professoras } \\
\text { de enfermagem e os saberes }\end{array}$ & REBEn & 2007 & Brasil \\
\hline CINAHL & On the Way to Learning & $\begin{array}{l}\text { MEDSURG } \\
\text { Nursing }\end{array}$ & 2009 & EUA \\
\hline CINAHL & $\begin{array}{l}\text { Factors Related to Successful Teaching } \\
\text { by } \\
\text { Outstanding Professors: An Interpretive }\end{array}$ & $\begin{array}{l}\text { Journal of Nursing } \\
\text { Education }\end{array}$ & 2009 & EUA \\
\hline MEDLINE & $\begin{array}{l}\text { O dito e o não-dito do ser-docente- } \\
\text { enfermeiro/a na compreensão da } \\
\text { sensibilidade. }\end{array}$ & REBEn & 2008 & Brasil \\
\hline MEDLINE & $\begin{array}{l}\text { Formação do enfermeiro: características } \\
\text { do professor e o sucesso escolar }\end{array}$ & REBEn & 2006 & Portugal \\
\hline MEDLINE & $\begin{array}{l}\text { Reflexões sobre competência docente } \\
\text { no ensino de enfermagem }\end{array}$ & $\begin{array}{l}\text { Rev Esc Enferm } \\
\text { USP }\end{array}$ & 2007 & Brasil \\
\hline SCIELO & $\begin{array}{l}\text { Prazer e sofrimento no processo de } \\
\text { trabalho do enfermeiro docente. }\end{array}$ & $\begin{array}{l}\text { Rev. da Escola } \\
\text { Enfermagem da } \\
\text { USP. }\end{array}$ & 2009 & Brasil \\
\hline SCIELO & $\begin{array}{l}\text { Incidentes críticos do processo ensino- } \\
\text { aprendizagem do curso de graduação } \\
\text { em enfermagem, segundo a percepção } \\
\text { de alunos e docentes }\end{array}$ & $\begin{array}{l}\text { Rev. Latino } \\
\text { america de } \\
\text { Enfermagem }\end{array}$ & 2006 & Brasil \\
\hline SCIELO & $\begin{array}{l}\text { O significado de competência para o } \\
\text { docente de administração em } \\
\text { enfermagem }\end{array}$ & $\begin{array}{l}\text { Rev. da Escola } \\
\text { Enfermagem da } \\
\text { USP. }\end{array}$ & 2006 & Brasil \\
\hline
\end{tabular}




\begin{tabular}{|c|c|c|c|c|}
\hline SCIELO & $\begin{array}{l}\text { Enfermeiro professor: um diálogo com a } \\
\text { formação pedagógica }\end{array}$ & REBEn & 2006 & Brasil \\
\hline WILSON & $\begin{array}{l}\text { Vozes da experiência: Reflexões dos } \\
\text { enfermeiros educadores (Voices of } \\
\text { Experience: Reflections of Nurse } \\
\text { Educators) }\end{array}$ & $\begin{array}{l}\text { The Journal of } \\
\text { Continuing } \\
\text { Education in } \\
\text { Nursing }\end{array}$ & 2009 & USA \\
\hline WILSON & $\begin{array}{l}\text { Cumplicidade ao alcance: Sabedoria } \\
\text { Prática de Enfermeira Educadores } \\
\text { Clínicos (Knowing Within: Practice } \\
\text { Wisdom of Clinical Nurse Educators) }\end{array}$ & $\begin{array}{c}\text { Journal of Nursing } \\
\text { Education }\end{array}$ & 2006 & USA \\
\hline WILSON & $\begin{array}{l}\text { Faculty Perceptions of Critical Thinking } \\
\text { in Student Clinical Experiences }\end{array}$ & $\begin{array}{c}\text { Journal of Nursing } \\
\text { Education }\end{array}$ & 2005 & USA \\
\hline
\end{tabular}

Fuente: Bases de datos LILACS, CINAHL, MEDLINE, SCIELO, WILSON, en el período de abril a junio, SC, 2010.

En la siguiente tabla se muestran los significados que los docentes atribuyen a la práctica pedagógica identificados en los artículos captados para el estudio:

\begin{tabular}{c|l}
\hline $\begin{array}{c}\text { No } \\
\text { Artículo }\end{array}$ & \multicolumn{1}{c}{ Significados encontrados } \\
\hline 1 & $\begin{array}{l}\text { Sentimiento de falta de preparación para ser profesor, así como la falta de } \\
\text { bases pedagógicas. } \\
\text { Inmadurez. } \\
\text { Descontento relacionado con las instituciones de enseñanza, con los } \\
\text { recursos por ellas disponibilizados. } \\
\text { Necesidad de valorización. } \\
\text { Necesidad de capacitación ofrecida por las instituciones. } \\
\text { Satisfacción en la busca de conocimientos, reflexión acerca de su práctica y } \\
\text { experiencias. }\end{array}$ \\
\hline 3 & $\begin{array}{l}\text { Tener experiencia en la profesión de enfermero, conocimientos previos } \\
\text { sobre determinado asunto, y creatividad en las situaciones cotidianas. } \\
\text { Ser innovador buscando prácticas pedagógicas innovadoras. }\end{array}$ \\
\hline $\begin{array}{l}\text { Saberes relacionados con la propia profesión y la práctica pedagógica, o } \\
\text { sea, con capacitaciones dirigidas a la carrera docente. } \\
\text { Seguridad que es adquirida a través de experiencia en la clínica y con el }\end{array}$ \\
\hline
\end{tabular}




\begin{tabular}{|c|c|}
\hline & $\begin{array}{l}\text { tiempo. } \\
\text { Placer en aprender en el cotidiano. }\end{array}$ \\
\hline 4 & $\begin{array}{l}\text { La participación del profesor en el proceso educativo, en la perspectiva de } \\
\text { ser aprendiz junto con el alumno. } \\
\text { Sentir que el aprender-enseñar es significativo para ambas partes del } \\
\text { proceso educativo. } \\
\text { Adquirir experiencia y realizaciones. } \\
\text { Sentimentos combinados de alegria, tristeza, miedo, compasión y } \\
\text { exuberancia. } \\
\text { Sentimento de igualdad entre alumno y profesor, en lo que se refiere al } \\
\text { status de aprendizaje. } \\
\text { Provocativo cuando invita al alumno a pensar y abre el camino del } \\
\text { aprendizaje. }\end{array}$ \\
\hline 5 & $\begin{array}{l}\text { Escasa preparación, atendiendo a que la carrera de profesor de enfermería } \\
\text { precisa ser preparada. } \\
\text { Estar presente en el proceso de aprendizaje del alumno, promoviendo el } \\
\text { aprendizaje. } \\
\text { Entusiasmo, desafío y gusto por enseñar. }\end{array}$ \\
\hline 6 & $\begin{array}{l}\text { Sensibilidad, solidaridad y responsabilidad. } \\
\text { Vivencia en el mundo de la vida y de la práctica pedagógica. } \\
\text { Proyectarse para el otro y para el mundo. } \\
\text { Tener libertad para cambiar. } \\
\text { Tener capacidad de ayudar al alumno a reflexionar. } \\
\text { Conseguir reaprender a observar el mundo de la vida. } \\
\text { Tener buenas relaciones e interacciones. } \\
\text { Hacer cambios en el proceso educativo adaptando las estrategias a cada } \\
\text { situación presentada. }\end{array}$ \\
\hline 7 & $\begin{array}{l}\text { Tener buenas relaciones con los alumnos. } \\
\text { Demostrar seguridad en lo que hace. } \\
\text { Estar disponible e implicado. } \\
\text { Gustar de lo que hace. } \\
\text { Capacidad de participar a los alumnos en el proceso de aprendizaje. }\end{array}$ \\
\hline 8 & $\begin{array}{l}\text { Tener competencia reflexiva construida a lo largo de la trayectoria personal y } \\
\text { profesional. } \\
\text { Expandirse más allá de su área técnica de formación, invadiendo espacios }\end{array}$ \\
\hline
\end{tabular}




\begin{tabular}{|c|c|}
\hline & $\begin{array}{l}\text { sociales de las relaciones interpersonales. } \\
\text { Construir a través de capacitación el perfil profesional de enfermería } \\
\text { orientado a la docencia. }\end{array}$ \\
\hline 9 & $\begin{array}{l}\text { Gusto por la interacción docente-alumno, en el compromiso con las } \\
\text { actividades didáctico-pedagógicas y en el compromiso con la formación } \\
\text { profesional. } \\
\text { Sufrimiento en lo que se refiere a desarticulación entre el empeño del } \\
\text { docente y del alumno, marcado por desinterés o falta de compromiso de los } \\
\text { discentes. }\end{array}$ \\
\hline 10 & $\begin{array}{l}\text { Aspectos negativos relacionados con la interacción con el grupo. } \\
\text { Aspecto positivo en la cooperación de los alumnos para ayudar a los demás. }\end{array}$ \\
\hline 11 & $\begin{array}{l}\text { Saber hacer en el sentido de conocer y transmitir conocimientos, gerenciar } \\
\text { el proceso de enseñanza-aprendizaje y las necesidades de integrar } \\
\text { contenidos en otras disciplinas. } \\
\text { Sensibilidad como competencia. }\end{array}$ \\
\hline 12 & $\begin{array}{l}\text { Tener dominio del área pedagógica. } \\
\text { Poder ser un docente reflexivo, como una forma de transformación. } \\
\text { Compromiso con la propia práctica. }\end{array}$ \\
\hline 13 & $\begin{array}{l}\text { Apasionarse por lo que hace; ser armonioso; invertir en las relaciones y } \\
\text { creer en sí mismo y en los otros. } \\
\text { Ser flexible y tener sentido del humor. } \\
\text { Motivación personal para ser un enfermero educador. } \\
\text { Tener compromiso con la formación de nuevos profesionales, siendo este el } \\
\text { gran desafío de la práctica docente. }\end{array}$ \\
\hline 14 & $\begin{array}{l}\text { Demostrar empeño, dinamismo y confianza en entrar en la situación } \\
\text { inesperada. } \\
\text { Los cuidados deben caracterizarse siempre por la conciencia de las } \\
\text { competencias básicas de prácticas seguras y el conocimento de las } \\
\text { responsabilidades sociales y morales de las enfermeras. } \\
\text { Ser un educador sintonizado con la diferencia, reflexionando y respondiendo } \\
\text { a las situaciones de forma moral y éticamente, y que pone de manifiesto el } \\
\text { compromiso de los educadores y del saber. }\end{array}$ \\
\hline 15 & $\begin{array}{l}\text { El significado está en la efectividad de la enseñanza a través de la cognición } \\
\text { del pensamiento crítico. }\end{array}$ \\
\hline
\end{tabular}

Fuente: Artículos científicos captados en las bases LILACS, CINAHL, MEDLINE, SCIELO, WILSON, en el período de abril a junio, SC, 2010. 
En la Tabla se percibe que los significados más citados son los que se refieren a capacitación de los profesores, experiencia profesional, en invertir y estar motivado para la carrera de profesor, gusto por lo que hace y estar comprometido con la enseñanza aprendizaje.

\section{DISCUSIÓN}

La revisión integrativa es considerada un tipo de investigación que presenta un amplio enfoque metodológico, donde se refieren las revisiones, permitiendo la inclusión de estudios experimentales y no experimentales para una comprensión completa del fenómeno analizado y que combina también datos de la literatura teórica y empírica ${ }^{(6)}$. Por eso permite que sean evidenciadas las lagunas de conocimiento existentes en la temática abordada.

En esta revisión los resultados fueron variados en lo que se refiere al significado de la práctica pedagógica para el docente en enfermería, se encontraron tanto aspectos positivos como negativos relacionados con la práctica docente, sin embargo destaca la discreta productividad de estudios relacionada con esta temática, teniendo en cuenta la importancia del profesor en cualquier esfera del proceso de enseñanza-aprendizaje, notando en ocasiones la falta de valorización de este profesional, lo que quedó evidenciado en diversos artículos representado por la necesidad de capacitación y de preparación del enfermero que desea seguir la carrera docente. Los aspectos de la formación del profesor reflexivo en enfermería es una preocupación constante, pues además de estar fundamentada en las cuestiones legales de cumplimiento de las directrices curriculares, está en el compromiso de formar profesionales competentes y ciudadanos que puedan contribuir a la mejora de la profesión, así hay una urgencia de capacitar enfermeros-profesores reflexivos para la práctica docente a través del incentivo de estudios de bases teóricas y metodológicas de la educación em enfermería ${ }^{(7)}$.

Se observó la necesidad de situar a estos profesionales en algún referencial teórico acerca de la educación en enfermería, atendiendo a los avances sobre las prácticas basadas en evidencias, y las estrategias de enseñanza-aprendizaje orientadas a la reflexión y la acción. La propuesta de articular la educación y la salud son desafíos, así como crear y proporcionar interfaces entre distintos saberes y poderes, pudiendo caracterizarse como una excepcional oportunidad para la profundización en los diálogos disciplinares generando alternativas metodológicas y, sobre todo, la renovación de conocimientos y prácticas en la salud ${ }^{(8)}$.

Los profesores de enfermería evidenciados en estos estudios, buscan en sus cotidianos mejorar sus estudios a fin de mejorar sus prácticas de enseñanza-aprendizaje, percibiendo que en su día a día se encuentran con situaciones inesperadas, que precisan ser competentes en la articulación de la práctica de ser-enfermero, de la práctica pedagógica y de la práctica docente para que puedan abrir posibilidades en la enseñanza, a fin de sugerir caminos en la resolución de los problemas presentados en el transcurrir del proceso de enseñanza-aprendizaje. Este tipo de actitud se configura en el pensamiento crítico-reflexivo por parte del enfermero-profesor que asume el papel de mediador del aprendizaje a ser construida por los sujetos de la práxis educativa, con miras a la autonomía en el proceso de aprender a aprender, reflexionando sobre la realidad en que está viviendo, estimulando el pensamiento crítico-reflexivo, y llevándolos a buscar alternativas de solución para los problemas encontrados, y transformando la propia realidad ${ }^{(9)}$. 
El enseñar y aprender no se configura como tarea fácil para los educadores frente al mundo globalizado donde la velocidad de informaciones y avances tecno-científicos constituye un desafío. Este desafío se hace sin embargo más complejo al enseñar a futuros profesionales el cuidado, desafío del enfermero-profesor, que precisa estar provisto de competencias que van más allá de la preparación de una clase, o de las paredes del aula, visto que el enfermero-profesor constantemente se ve en situaciones distintas en su trabajo cotidiano. Estas situaciones implican no sólo al profesor y al alumno, sino a otros sujetos, tales como pacientes, familias, miembros del equipo de salud. Estas situaciones pueden ser experienciadas con alegría, angustia y pesar.

Aunque ser enfermero profesor sea una tarea compleja, se observa cada vez más el interés en buscar nuevas alternativas en la enseñanza de enfermería denotando la preocupación con el proceso de formación de profesores y en este sentido formar ciudadanos competentes que puedan contribuir para la profesión ${ }^{(10)}$.

Se identificó, en este estudio que la baja demanda de estudios dirigidos al existencialismo del profesor, en este caso de enfermería, se configura en una laguna del conocimiento. Así, se refuerza la necesidad constante de repensar las prácticas pedagógicas y la práctica docente, a través de espacios para la capacitación de enfermeros-profesores, que pueden ser vivenciados como espacios de socialización de los saberes en enfermería, de los intercambios de experiencias y, ¿por qué no?, espacios para retomar la verdadera esencia del ser-enfermero-profesor.

\section{REFERENCIAS}

1. Reibnitz KS, Prado ML. Inovação e educação em Enfermagem. Ed. Cidade Futura: Florianópolis, SC. 2006.

2. Faria JIL, Casagrande LDR. A educação para o século XXI... e a formação do professor reflexivo na enfermagem Rev Latino-am Enfermagem , 2004; 2(5):821-7

3. Freire P. Pedagogia da Autonomia: Saberes necessários à pratica educativa. 33 ed. Editora Paz e Terra: São Paulo. 2006.

4. Ganong LH. Integrative reviews of nursing research. Res Nurs Health, 1987;10(1):1-11.

5. Polit DF, Beck CT, Hungler BP. Fundamentos de pesquisa em enfermagem: métodos, avaliação e utilização. 5a ed. Porto Alegre (RS): Artmed, 2004.

6. Souza MT, Silva MD, Carvalho R. Revisão integrativa: o que é e como fazer. Rev. Einstein, 2010; 8(1 Pt 1):102-6.

7. Faria JIL, Casagrande LDR. A educação para o século XXI... Rev Latino-am Enfermagem, 2004; 12(5):821-7 www.eerp.usp.br/rlae

8. Monteiro S, Vargas E. (Orgs.). Educação, comunicação e tecnologia educacional: interfaces com o campo da saúde. Rio de Janeiro: Editora Fiocruz, 2006. 252p.

9. Valente GS, Viana LO. O pensamento crítico-reflexivo no ensino da pesquisa em enfermagem: um desafio para o professor!, Enfermería Global, 2007; № 10.

10. Macedo-Costa KN, De Almeida Rebouças CB,Freitas da Silva GR, Markert W. Enfermero-docente: aspectos interrelacionados con su formación, Rev. Enfermería Global, 2010; $\mathrm{N}^{\mathrm{O}} 19: 1-14$. 
